\title{
Delay-dependent Exponential Stability of Neutral Stochastic Delay Systems
}

\author{
Lirong Huang and Xuerong Mao
}

\begin{abstract}
This paper studies stability of neutral stochastic delay systems by linear matrix inequality (LMI) approach. Delaydependent criterion for exponential stability is presented and numerical examples are conducted to verify the effectiveness of the proposed method.
\end{abstract}

Index Terms - stochastic systems, neutral systems, time delay, exponential stability, LMIs.

\section{INTRODUCTION}

Many dynamical systems are described with neutral functional differential equations that include neutral delay differential equations. These systems are called neutral-type systems or neutral systems. Motivated by chemical engineering systems as well as theory of aero elasticity, studies on deterministic neutral systems have been of research interest over the past decades (see, e.g., [3]-[11]). As stochastic modelling has come to play an important role in many branches of science and industry, neutral stochastic delay systems have been intensively studied over recent year (see, e.g., [10]-[17]). Mao ([14]-[17]) initiated the study of exponential stability of neutral stochastic functional equations, developed the Razumikhin-type theorems further for exponential stability of neutral stochastic functional equations and studied asymptotic properties of neutral stochastic delay differential equations. More recently, Luo et al. ([12]) proposed new criteria on exponential stability of neutral stochastic delay differential equations while Chen et al. ([2]) studied delay-dependent stability of neutral stochastic delay systems. However, the stability result in ([2]) employed an assumption on the difference operator matrix, which is also assumed in other results (see, e.g., [4] and [18]) but may be restrictive in many cases (see Examples 1 and 2). As is known, delay-independent results may be conservative when the size of time delay is small. This paper studies problem of delaydependent stability of neutral stochastic delay systems. An exponential stability criterion is established by linear matrix inequality (LMI) approach. Numerical examples are conducted to verify the effectiveness of our proposed method.

\section{PRoblem StATEMENT}

Throughout the paper, unless otherwise specified, we will employ the following notation. Let $\left(\Omega, \mathcal{F},\left\{\mathcal{F}_{t}\right\}_{t \geq 0}, \mathbb{P}\right)$ be a complete probability space with a natural filtration $\left\{\mathcal{F}_{t}\right\}_{t \geq 0}$

Lirong Huang and Xuerong Mao are with Department of Statistics and Modelling Science, University of Strathclyde, Glasgow G1 1XH, UK (e-mail: lirong@stams.strath.ac.uk) and $\mathbb{E}[\cdot]$ be the expectation operator with respect to the probability measure. Let $w(t)$ be a scalar Brownian motion defined on the probability space. If $A$ is a vector or matrix, its transpose is denoted by $A^{T}$. If $P$ is a square matrix, $P>0(P<0)$ means that $P$ is a symmetric positive (negative) definite matrix of appropriate dimensions while $P \geq 0(P \leq 0)$ is a symmetric positive (negative) semidefinite matrix. $I$ stands for the identity matrix of appropriate dimensions. Denote by $\lambda_{M}(\cdot)$ and $\lambda_{m}(\cdot)$ the maximum and minimum eigenvalue of a matrix respectively. Let $|\cdot|$ denote the Euclidean norm of a vector and its induced norm of a matrix. Unless explicitly specified, matrices are assumed to have real entries and compatible dimensions. Let $h \geq 0$ and $C\left([-h, 0] ; R^{n}\right)$ denote the family of all continuous $R^{n}$-valued functions $\varphi$ on $[-h, 0]$ with the norm $\|\varphi\|=\sup \{|\varphi(\theta)|$ : $-h \leq \theta \leq 0\}$. Let $C_{\mathcal{F}_{0}}^{b}\left([-h, 0] ; R^{n}\right)$ be the family of all $\mathcal{F}_{0^{-}}$ measurable bounded $C\left([-h, 0] ; R^{n}\right)$-valued random variables $\xi=\{\xi(\theta):-h \leq \theta \leq 0\}$.

Let us consider an $n$-dimensional neutral stochastic delay system

$$
\begin{aligned}
& \mathrm{d}\left[x(t)-C x\left(t-h_{1}\right)\right] \\
& =\left[A_{0} x(t)+A_{1} x\left(t-h_{1}\right)+A_{2} x\left(t-h_{2}\right)\right] \mathrm{d} t \\
& \quad+\left[H_{0} x(t)+H_{1} x\left(t-h_{1}\right)+H_{2} x\left(t-h_{2}\right)\right] \mathrm{d} w(t)(1)
\end{aligned}
$$

on $t \geq 0$ with initial data $x_{0}=\{x(\theta):-h \leq \theta \leq 0\}=$ $\xi \in C_{\mathcal{F}_{0}}^{b}\left([-h, 0] ; R^{n}\right)$, where $x(t) \in R^{n}$ is the state vector; positive scalar constants $h_{1}, h_{2}$ are time delays of the system and $h=\max \left\{h_{1}, h_{2}\right\} ; C, A_{i}$ and $H_{i}, i=0,1,2$, are known matrices.

Denote

$$
\begin{aligned}
& f(t)=A_{0} x(t)+A_{1} x\left(t-h_{1}\right)+A_{2} x\left(t-h_{2}\right), \\
& g(t)=H_{0} x(t)+H_{1} x\left(t-h_{1}\right)+H_{2} x\left(t-h_{2}\right)
\end{aligned}
$$

for all $t \geq 0$. One can observe that

$$
|f(t)|^{2} \leq K_{f}\left\|x_{t}\right\|^{2}, \quad|g(t)|^{2} \leq K_{g}\left\|x_{t}\right\|^{2}
$$

for all $t \geq 0$, where $x_{t}=\{x(t+\theta):-h \leq \theta \leq 0\}$, $K_{f}=3 \sum_{i=0}^{2}\left|A_{i}\right|^{2}$ and $K_{g}=3 \sum_{i=0}^{2}\left|H_{i}\right|^{2}$. This implies that both $f(\varphi, t)$ and $g(\varphi, t)$ satisfy the local Lipschitz condition and the linear growth condition. It is easy to verify, by the way of induction proposed in the proof of Theorem 3.1, p208, [16], that there exists a unique continuous solution denoted by $x(t ; \xi)$ to neutral stochastic delay differential equation (1).

The objective of this paper is to establish sufficient conditions for robust exponential stability of system (1). It should be pointed out that, for simplicity only, we do not consider 
uncertainties in our models. The proposed method can be easily extended to those cases with norm-bounded uncertainties in parameters $A_{i}$ and $H_{i}$. The method can also be applied to systems with multiple and distributed delays.

At the end of this section, let us introduce the following definitions and lemmas that are useful for the development of our results.

Definition 1: ([16]) The neutral stochastic delay system (1) is said to be exponentially stable in mean square if there is a positive constant $\lambda$ such that

$$
\limsup _{t \rightarrow \infty} \frac{1}{t} \log \mathbb{E}|x(t ; \xi)|^{2} \leq-\lambda .
$$

Definition 2: ([16]) The neutral stochastic delay system (1) is said to be almost surely exponentially stable if there is a positive constant $\lambda$ such that

$$
\limsup _{t \rightarrow \infty} \frac{1}{t} \log |x(t ; \xi)| \leq-\lambda .
$$

Lemma 1: ([20]) For any constant matrix $M \in R^{q \times l}$, inequality

$$
2 u^{T} M v \leq r u^{T} M G M^{T} u+\frac{1}{r} v^{T} G^{-1} v, \quad u \in R^{q}, v \in R^{l}
$$

holds for any pair of symmetric positive definite matrix $G \in$ $R^{l \times l}$ and positive number $r>0$.

Lemma 2: ([6]) For any pair of symmetric positive definite constant matrix $G \in R^{l \times l}$ and scalar $r>0$, if there exists a vector function $v:[0, r] \rightarrow R^{l}$ such that integrals $\int_{0}^{r} v^{T}(s) G v(s) \mathrm{d} s$ and $\int_{0}^{r} v(s) \mathrm{d} s$ are well defined, then the following inequality holds

$$
r \int_{0}^{r} v^{T}(s) G v(s) \mathrm{d} s \geq\left(\int_{0}^{r} v(s) \mathrm{d} s\right)^{T} G\left(\int_{0}^{r} v(s) \mathrm{d} s\right) .
$$

\section{Delay-DEPEndEnt EXPONENTIAL STABILITy}

Delay-dependent stability of neutral deterministic delay systems has been intensively studied over recent years (see, e.g., [3]-[5], [8], [11], [18]). However, relatively little is known about delay-dependent stability of neutral stochastic delay systems. Denote $\bar{A}_{0}=A_{0}, \bar{A}_{1}=A_{0} C+A_{1}, \bar{A}_{2}=A_{2}$, $\bar{H}_{0}=H_{0}, \bar{H}_{1}=H_{0} C+H_{1}, \bar{H}_{2}=H_{2}, \bar{A}=\sum_{i=0}^{2} \bar{A}_{i}$ and $\bar{H}=\sum_{i=0}^{2} \bar{H}_{i}$. Sufficient conditions for delay-dependent exponential stability of system (1) are proposed as follows.

Theorem 1: The neutral stochastic delay system (1) is mean-square exponentially stable and is also almost surely exponentially stable provided that there exist matrices $P_{11}>$ $0, Q_{k}>0, R_{k}>0, S>0, T_{k}>0, P_{21}, P_{22}, P_{23}, P_{31}, P_{32}$, $P_{33}$ and $k=1,2$ such that LMI (6) (on the top of next page) holds,

where

$$
\begin{aligned}
& \Gamma_{11}=P_{21}^{T} \bar{A}+\bar{A}^{T} P_{21}+P_{31}^{T} \bar{H}+\bar{H}^{T} P_{31}+S+T_{1}+T_{2}, \\
& \Gamma_{12}=\bar{A}^{T} P_{22}+\bar{H}^{T} P_{32}+P_{11}-P_{21}^{T}, \\
& \Gamma_{13}=\bar{A}^{T} P_{23}+\bar{H}^{T} P_{33}-P_{31}^{T}, \quad \Gamma_{18}=\left(S+T_{1}+T_{2}\right) C, \\
& \Gamma_{22}=-P_{22}^{T}-P_{22}+h_{1} Q_{1}+h_{2} Q_{2}, \quad \Gamma_{23}=-P_{23}-P_{32}^{T},
\end{aligned}
$$

$$
\begin{aligned}
& \Gamma_{33}=-P_{33}^{T}-P_{33}+P_{11}+h_{1} R_{1}+h_{2} R_{2}, \\
& \Gamma_{88}=-S+C^{T}\left(S+T_{1}+T_{2}\right) C, \\
& L_{11}=P_{21}^{T} \bar{A}_{1}+P_{31}^{T} \bar{H}_{1}, \quad L_{12}=P_{21}^{T} \bar{A}_{2}+P_{31}^{T} \bar{H}_{2}, \\
& L_{21}=P_{22}^{T} \bar{A}_{1}+P_{32}^{T} \bar{H}_{1}, \quad L_{22}=P_{22}^{T} \bar{A}_{2}+P_{32}^{T} \bar{H}_{2}, \\
& L_{31}=P_{23}^{T} \bar{A}_{1}+P_{33}^{T} \bar{H}_{1}, \quad L_{32}=P_{23}^{T} \bar{A}_{2}+P_{33}^{T} \bar{H}_{2},
\end{aligned}
$$

and entries denoted by $*$ can be readily inferred from symmetry of the matrix.

Proof: To simplify the expression, we define

$$
\eta(t)=x(t)-C x\left(t-h_{1}\right)
$$

for all $t \geq 0$. With notations (2) and (7), we can rewrite the unforced system (1) as

$$
\mathrm{d} \eta(t)=f(t) \mathrm{d} t+g(t) \mathrm{d} w(t)
$$

on $t \geq 0$ with initial data $\xi$.

So we have

$$
\eta\left(t_{2}\right)-\eta\left(t_{1}\right)=\int_{t_{1}}^{t_{2}} f(s) \mathrm{d} s+g(s) \mathrm{d} w(s)
$$

for all $t_{2} \geq t_{1} \geq 0$.

By (2) and (9), we can observe that

$$
\begin{aligned}
f(t)= & \sum_{i=0}^{2} \bar{A}_{i} \eta(t)-\sum_{i=1}^{2} \bar{A}_{i}\left[\eta(t)-\eta\left(t-h_{i}\right)\right] \\
& +\sum_{i=1}^{2} \bar{A}_{i} C x\left(t-h_{1}-h_{i}\right) \\
= & \bar{A} \eta(t)-\sum_{i=1}^{2} \bar{A}_{i} \int_{t-h_{i}}^{t} f(s) \mathrm{d} s+g(s) \mathrm{d} w(s) \\
& +\sum_{i=1}^{2} \bar{A}_{i} C x\left(t-h_{1}-h_{i}\right) \\
g(t)= & \bar{H} \eta(t)-\sum_{i=1}^{2} \bar{H}_{i} \int_{t-h_{i}}^{t} f(s) \mathrm{d} s+g(s) \mathrm{d} w(s) \\
& +\sum_{i=1}^{2} \bar{H}_{i} C x\left(t-h_{1}-h_{i}\right)
\end{aligned}
$$

for all $t \geq h$. Choose a Lyapunov-Krasovskii functional candidate for system (8) as follows

$$
V(t)=\sum_{j=1}^{5} V_{j}(t), \quad t \geq h
$$

where

$$
\begin{aligned}
& V_{1}(t)=\eta^{T}(t) P_{11} \eta(t) \\
& V_{2}(t)=\sum_{i=1}^{2} \int_{t-h_{i}}^{t}\left(s-t+h_{i}\right) f^{T}(s) Q_{i} f(s) \mathrm{d} s \\
& V_{3}(t)=\sum_{i=1}^{2} \int_{t-h_{i}}^{t}\left(s-t+h_{i}\right) g^{T}(s) R_{i} g(s) \mathrm{d} s \\
& V_{4}(t)=\int_{t-h_{1}}^{t} x^{T}(s) S x(s) \mathrm{d} s \\
& V_{5}(t)=\sum_{i=1}^{2} \int_{t-h_{1}-h_{i}}^{t} x^{T}(s) T_{i} x(s) \mathrm{d} s .
\end{aligned}
$$




$$
\Gamma=\left[\begin{array}{cccccccccc}
\Gamma_{11} & \Gamma_{12} & \Gamma_{13} & h_{1} L_{11} & h_{2} L_{12} & L_{11} & L_{12} & \Gamma_{18} & L_{11} C & L_{12} C \\
* & \Gamma_{22} & \Gamma_{23} & h_{1} L_{21} & h_{2} L_{22} & L_{21} & L_{22} & 0 & L_{21} C & L_{22} C \\
* & * & \Gamma_{33} & h_{1} L_{31} & h_{2} L_{32} & L_{31} & L_{32} & 0 & L_{31} C & L_{32} C \\
* & * & * & -h_{1} Q_{1} & 0 & 0 & 0 & 0 & 0 & 0 \\
* & * & * & 0 & -h_{2} Q_{2} & 0 & 0 & 0 & 0 & 0 \\
* & * & * & 0 & 0 & -R_{1} & 0 & 0 & 0 & 0 \\
* & * & * & 0 & 0 & 0 & -R_{2} & 0 & 0 & 0 \\
* & 0 & 0 & 0 & 0 & 0 & 0 & \Gamma_{88} & 0 & 0 \\
* & * & * & 0 & 0 & 0 & 0 & 0 & -T_{1} & 0 \\
* & * & * & 0 & 0 & 0 & 0 & 0 & 0 & -T_{2}
\end{array}\right]<0
$$

By Itô's lemma, we have

$$
\mathrm{d} V(t)=\mathcal{L} V(t) \mathrm{d} t+\sigma(t) \mathrm{d} w(t)
$$

where

$$
\begin{aligned}
\mathcal{L} V(t)= & \sum_{j=1}^{5} \mathcal{L} V_{j}(t)=2 \eta^{T}(t) P_{11} f(t)+g^{T}(t) P_{11} g(t) \\
& +\sum_{j=2}^{5} \dot{V}_{j}(t), \\
\sigma(t)= & 2 \eta^{T}(t) P_{11} g(t) .
\end{aligned}
$$

Denote

$$
y(t)=\left[\begin{array}{l}
\eta(t) \\
f(t) \\
g(t)
\end{array}\right] \text { and } P=\left[\begin{array}{ccc}
P_{11} & 0 & 0 \\
P_{21} & P_{22} & P_{23} \\
P_{31} & P_{32} & P_{33}
\end{array}\right] .
$$

By equalities (10) and (11), we have

$$
\begin{aligned}
& 2 \eta^{T}(t) P_{11} f(t) \\
& =y^{T}(t)\left(P^{T} A+A^{T} P\right) y(t)-2 y^{T}(t) \sum_{i=1}^{2} P^{T}\left[\begin{array}{ll}
0 & \bar{A}_{i}^{T}
\end{array}\right. \\
& \cdot\left(\int_{t-h_{i}}^{t} f(s) \mathrm{d} s+g(s) \mathrm{d} w(s)+C x\left(t-h_{1}-h_{i}\right)\right)
\end{aligned}
$$

where

$$
\begin{aligned}
& A=\left[\begin{array}{ccc}
0 & I & 0 \\
\bar{A} & -I & 0 \\
\bar{H} & 0 & -I
\end{array}\right], \\
& P^{T} A+A^{T} P=\left[\begin{array}{ccc}
P_{A 1} & P_{A 2} & P_{A 3} \\
* & -P_{22}^{T}-P_{22} & -P_{32}^{T}-P_{23} \\
* & * & -P_{33}^{T}-P_{33}
\end{array}\right]
\end{aligned}
$$

with $P_{A 1}=P_{21}^{T} \bar{A}+\bar{A}^{T} P_{21}+P_{31}^{T} \bar{H}+\bar{H}^{T} P_{31}, P_{A 2}=\bar{A}^{T} P_{22}+$ $\bar{H}^{T} P_{32}+P_{11}-P_{21}^{T}, P_{A 3}=\bar{A}^{T} P_{23}+\bar{H}^{T} P_{33}-P_{31}^{T}$ and $P^{T}\left[\begin{array}{lll}0 & \bar{A}_{i}^{T} & \bar{H}_{i}^{T}\end{array}\right]^{T}=\left[\begin{array}{lll}L_{1 i}^{T} & L_{2 i}^{T} & L_{3 i}^{T}\end{array}\right]^{T}$ for $i=1,2$.

Direct computations with Lemma 2 and equation (7) give

$$
\begin{aligned}
\dot{V}_{2}(t) \leq & \sum_{i=1}^{2}\left[f^{T}(t) h_{i} Q_{i} f(t)-\int_{t-h_{i}}^{t} \frac{1}{h_{i}} f^{T}(s) \mathrm{d} s \cdot\left(h_{i} Q_{i}\right)\right. \\
& \left.\cdot \int_{t-h_{i}}^{t} \frac{1}{h_{i}} f(s) \mathrm{d} s\right], \\
\dot{V}_{3}(t)= & \sum_{i=1}^{2}\left[g^{T}(t) h_{i} R_{i} g(t)-\int_{t-h_{i}}^{t} g^{T}(s) R_{i} g(s) \mathrm{d} s\right],
\end{aligned}
$$

$$
\dot{V}_{4}(t)=\left[\begin{array}{c}
\eta(t) \\
x\left(t-h_{1}\right)
\end{array}\right]^{T}\left[\begin{array}{cc}
S & S C \\
C^{T} S & -S+C^{T} S C
\end{array}\right]\left[\begin{array}{c}
\eta(t) \\
x\left(t-h_{1}\right)
\end{array}\right],
$$

$$
\begin{aligned}
\dot{V}_{5}(t)= & \sum_{i=1}^{2}\left[\begin{array}{c}
\eta(t) \\
x\left(t-h_{1}\right) \\
x\left(t-h_{1}-h_{i}\right)
\end{array}\right]^{T}\left[\begin{array}{ccc}
T_{i} & T_{i} C & 0 \\
C^{T} T_{i} & C^{T} T_{i} C & 0 \\
0 & 0 & -T_{i}
\end{array}\right] \\
& \cdot\left[\begin{array}{c}
\eta(t) \\
x\left(t-h_{1}\right) \\
x\left(t-h_{1}-h_{i}\right)
\end{array}\right]
\end{aligned}
$$

By isometry property, for $i=1,2$, we have

$$
\begin{aligned}
& \mathbb{E}\left[\int_{t-h_{i}}^{t} g^{T}(s) R_{i} g(s) \mathrm{d} s\right]=\int_{t-h_{i}}^{t} \mathbb{E}\left[g^{T}(s) R_{i} g(s)\right] \mathrm{d} s \\
& =\mathbb{E}\left[\int_{t-h_{i}}^{t} g^{T}(s) \mathrm{d} w(s) R_{i} \int_{t-h_{i}}^{t} g(s) \mathrm{d} w(s)\right] .
\end{aligned}
$$

Therefore, substituting inequalities (16)-(20) into (14) and taking expectation on the both sides of (14) yield

$$
\mathbb{E} \mathcal{L} V(t) \leq \mathbb{E}\left[z^{T}(t) \Gamma z(t)\right]
$$

where $z^{T}(t)=\left[\eta^{T}(t) f^{T}(t) g^{T}(t) \frac{1}{h_{1}} \int_{t-h_{1}}^{t} f^{T}(s) \mathrm{d} s\right.$ $\frac{1}{h_{2}} \int_{t-h_{2}}^{t} f^{T}(s) \mathrm{d} s \int_{t-h_{1}}^{t} g^{T}(s) \mathrm{d} w(s) \int_{t-h_{2}}^{t} g^{T}(s) \mathrm{d} w(s)$ $\left.x^{T}\left(t-h_{1}\right) x^{T}\left(t-2 h_{1}\right) x^{T}\left(t-h_{1}-h_{2}\right)\right]^{T}$.

By LMI (6), we have

$$
\mathbb{E} \mathcal{L} V(t) \leq-\lambda_{\Gamma} \mathbb{E}|z(t)|^{2} \leq-\lambda_{\Gamma} \mathbb{E}\left[|\eta(t)|^{2}+\left|x\left(t-h_{1}\right)\right|^{2}\right]
$$

with $\lambda_{\Gamma}=\lambda_{m}(-\Gamma)$ and

$$
C^{T} S C-S<0 .
$$

For any $\kappa \in(0,1)$, equation (7), inequalities (22)-(23) and Lemma 1 give

$$
\begin{aligned}
& \mathbb{E} \mathcal{L} V(t) \\
& \leq-(1-\kappa) \lambda_{\Gamma} \mathbb{E}|\eta(t)|^{2}-\kappa \lambda_{\Gamma}\left(\mathbb{E}|\eta(t)|^{2}+\frac{1}{\kappa} \mathbb{E}\left|x\left(t-h_{1}\right)\right|^{2}\right) \\
& \leq-(1-\kappa) \lambda_{\Gamma} \mathbb{E}|\eta(t)|^{2}-\frac{\kappa \lambda_{\Gamma}}{\lambda_{M}(S)} \mathbb{E}\left[\left(x(t)-C x\left(t-h_{1}\right)\right)^{T} S\right. \\
& \left.\cdot\left(x(t)-C x\left(t-h_{1}\right)\right)+\frac{1}{\kappa} x^{T}\left(t-h_{1}\right) S x\left(t-h_{1}\right)\right] \\
& \leq-(1-\kappa) \lambda_{\Gamma} \mathbb{E}|\eta(t)|^{2}-\frac{\kappa \lambda_{\Gamma}}{\lambda_{M}(S)} \mathbb{E}\left[x^{T}(t) S x(t)\right. \\
& \left.-2 x^{T}(t) S C x\left(t-h_{1}\right)+\frac{1+\kappa}{\kappa} x^{T}\left(t-h_{1}\right) C^{T} S C x\left(t-h_{1}\right)\right] \\
& \leq-(1-\kappa) \lambda_{\Gamma} \mathbb{E}|\eta(t)|^{2}-\frac{\kappa \lambda_{\Gamma} \lambda_{m}(S)}{(1+\kappa) \lambda_{M}(S)} \mathbb{E}|x(t)|^{2} . \\
& \leq-\lambda_{0} \mathbb{E}\left[|\eta(t)|^{2}+|x(t)|^{2}\right],
\end{aligned}
$$


where $\lambda_{0}=\min \left\{(1-\kappa) \lambda_{\Gamma}, \kappa \lambda_{\Gamma} \lambda_{m}(S)\left[(1+\kappa) \lambda_{M}(S)\right]^{-1}\right\}$ $>0$.

It is obvious from the definition of $V(t)$ that

$$
\alpha_{0}|\eta(t)|^{2} \leq V(t) \leq \alpha_{1}|\eta(t)|^{2}+\alpha_{2} \int_{t-2 h}^{t}|x(s)|^{2} \mathrm{~d} s,
$$

where $\alpha_{0}=\lambda_{m}\left(P_{11}\right), \quad \alpha_{1}=\lambda_{M}\left(P_{11}\right), \alpha_{2}=$ $\sum_{i=1}^{2} h_{i}\left[\lambda_{M}\left(Q_{i}\right) K_{f}+\lambda_{M}\left(R_{i}\right) K_{g}\right]+\lambda_{M}(S)+\sum_{i=1}^{2} \lambda_{M}\left(T_{i}\right)$. Choose $\varepsilon>0$ such that

$$
\max \left\{\varepsilon \alpha_{1}, 2 h \varepsilon \alpha_{2} e^{2 h \varepsilon}\right\} \leq \lambda_{0} \quad \text { and } \quad e^{2 h \varepsilon} C^{T} S C-S<0 .
$$

By Itô's lemma, we have

$\mathrm{d}\left[e^{\varepsilon s} V(s)\right]=e^{\varepsilon s}[\varepsilon V(s)+\mathcal{L} V(s)] \mathrm{d} s+e^{\varepsilon s} \sigma(s) \mathrm{d} w(s), \quad \forall s \geq 0$.

Let $t_{0}=h$, then integrating from $t_{0}$ to $t$ and taking expectation on (26) give

$$
\begin{array}{rl}
e^{\varepsilon t} & \mathbb{E} V(t)-e^{\varepsilon t_{0}} \mathbb{E} V\left(t_{0}\right) \\
= & \mathbb{E} \int_{t_{0}}^{t} e^{\varepsilon s}[\varepsilon V(s)+\mathcal{L} V(s)] \mathrm{d} s \\
\leq & \mathbb{E} \int_{t_{0}}^{t} e^{\varepsilon s}\left[\varepsilon \alpha_{1}|\eta(s)|^{2}+\varepsilon \alpha_{2} \int_{s-2 h}^{s}|x(v)|^{2} \mathrm{~d} v\right. \\
& \left.-\lambda_{0}\left(|\eta(s)|^{2}+|x(s)|^{2}\right)\right] \mathrm{d} s \\
\leq & \mathbb{E} \int_{t_{0}}^{t} e^{\varepsilon s}\left[\varepsilon \alpha_{2} \int_{s-2 h}^{s}|x(v)|^{2} \mathrm{~d} v-\lambda_{0}|x(s)|^{2}\right] \mathrm{d} s
\end{array}
$$

Since

$$
\begin{aligned}
& \int_{t_{0}}^{t} e^{\varepsilon s} \mathrm{~d} s \int_{s-2 h}^{s}|x(v)|^{2} \mathrm{~d} v \leq \int_{t_{0}-2 h}^{t}|x(v)|^{2} \mathrm{~d} v \int_{v}^{v+2 h} e^{\varepsilon s} \mathrm{~d} s \\
& \leq 2 h e^{2 h \varepsilon} \int_{t_{0}-2 h}^{t}|x(s)|^{2} e^{\varepsilon s} \mathrm{~d} s \\
& \leq 2 h e^{2 h \varepsilon} \int_{t_{0}}^{t}|x(s)|^{2} e^{\varepsilon s} \mathrm{~d} s+2 h e^{2 h \varepsilon} \int_{t_{0}-2 h}^{t_{0}}|x(s)|^{2} \mathrm{~d} s,
\end{aligned}
$$

it follows

$$
\alpha_{0} e^{\varepsilon t} \mathbb{E}|\eta(t)|^{2} \leq e^{\varepsilon t} \mathbb{E} V(t) \leq \alpha_{0} C_{h} \text { or } \mathbb{E}|\eta(t)|^{2} \leq C_{h} e^{-\varepsilon t},
$$

where $C_{h}=\alpha_{h} \sup _{-h \leq \theta \leq h} \mathbb{E}|x(\theta)|^{2}$ with $\alpha_{h}=\alpha_{0}^{-1} e^{\varepsilon h}\left[\alpha_{1}+\right.$ $\left.2 h \alpha_{2}\left(1+2 h \varepsilon e^{2 h \varepsilon}\right)\right] \geq 1$. Since neutral stochastic delay differential equation (1) has a unique continuous solution, $C_{h}$ is a nonnegative finite number for any $0 \leq h<\infty$.

Since $e^{2 \varepsilon h} C^{T} S C<S$, there exists a number $\mu \in(0,1)$ such that

$$
e^{2 \varepsilon h} C^{T} S C<\mu S<S .
$$

Note that $\eta^{T}(t) S \eta(t)=x^{T}(t) S x(t)-2 x^{T}(t) S C x\left(t-h_{1}\right)+$ $x^{T}\left(t-h_{1}\right) C^{T} S C x\left(t-h_{1}\right)$ for all $t \geq 0$. By Lemma 1 , we have

$$
\begin{aligned}
e^{\varepsilon t} x^{T}(t) S x(t) \leq & \frac{e^{\varepsilon t}}{1-\mu} \eta^{T}(t) S \eta(t) \\
& +\frac{e^{\varepsilon t}}{\mu} x^{T}\left(t-h_{1}\right) C^{T} S C x\left(t-h_{1}\right) .
\end{aligned}
$$

Let $\rho$ be any nonnegative real number. For all $0 \leq t \leq \rho$, we have

$$
\begin{array}{rl}
e^{\varepsilon t} & \mathbb{E}\left[x^{T}(t) S x(t)\right] \\
\leq & \frac{1}{1-\mu} \sup _{0 \leq t \leq \rho} \mathbb{E}\left[e^{\varepsilon t} \eta^{T}(t) S \eta(t)\right] \\
& +\frac{1}{\mu} \sup _{0 \leq t \leq \rho} \mathbb{E}\left[e^{\varepsilon t} x^{T}\left(t-h_{1}\right) C^{T} S C x\left(t-h_{1}\right)\right] \\
\leq & \frac{1}{1-\mu} \lambda_{M}(S) \sup _{0 \leq t \leq \rho} \mathbb{E}\left[e^{\varepsilon t}|\eta(t)|^{2}\right] \\
& +\frac{e^{\varepsilon h_{1}}}{\mu} \sup _{-h_{1} \leq t \leq \rho} \mathbb{E}\left[e^{\varepsilon t} x^{T}(t) C^{T} S C x(t)\right] \\
\leq & \frac{1}{1-\mu} \lambda_{M}(S) C_{h}+e^{-\varepsilon h} \sup _{-h \leq t \leq \rho}\left\{e^{\varepsilon t} \mathbb{E}\left[x^{T}(t) S x(t)\right]\right\} .
\end{array}
$$

But this holds for all $-h \leq t \leq \rho$. So

$$
\sup _{-h \leq t \leq \rho}\left\{e^{\varepsilon t} \mathbb{E}\left[x^{T}(t) S x(t)\right]\right\} \leq \frac{\lambda_{M}(S) C_{h}}{\left(1-e^{-\varepsilon h}\right)(1-\mu)} .
$$

Since $\rho$ is an arbitary nonnegative number, we have

$$
\mathbb{E}|x(t)|^{2} \leq \frac{\lambda_{M}(S) C_{h} e^{-\varepsilon t}}{\left(1-e^{-\varepsilon h}\right)(1-\mu) \lambda_{m}(S)}, \quad \forall t \geq-h .
$$

The mean-square exponential stability has been proven.

Now let us proceed to discuss the almost sure exponential stability. Let $\gamma \in(0, \varepsilon)$ be arbitrary. We claim that there is a finite positive number $t_{h}$ such that for all $t \geq t_{h}$

$$
|\eta(t)|^{2} \leq e^{-(\varepsilon-\gamma) t} \quad \text { a.s. }
$$

Therefore, for all $t \geq t_{h}$, inequality (30) implies

$$
\begin{aligned}
& e^{(\varepsilon-\gamma) t} x^{T}(t) S x(t) \\
& \leq \frac{\lambda_{M}(S) e^{(\varepsilon-\gamma) t}}{1-\mu}+\frac{e^{(\varepsilon-\gamma) t}}{\mu} x^{T}\left(t-h_{1}\right) C^{T} S C x\left(t-h_{1}\right) \text { a.s. }
\end{aligned}
$$

Using the similar reasoning as above and letting $\gamma \rightarrow 0$, we have $|x(t)|^{2} \leq \lambda_{M}(S) e^{-\varepsilon t}\left[\left(1-e^{-\varepsilon h}\right)(1-\mu) \lambda_{m}(S)\right]^{-1}$ a.s. for all $t \geq t_{h}-h$. This implies immediately

$$
\limsup _{t \rightarrow \infty} \frac{1}{t} \log |x(t)| \leq-\frac{\varepsilon}{2} \quad \text { a.s. }
$$

We complete the proof by showing that inequality (33) is true. Note that

$$
\mathbb{E}|f(t)|^{2} \leq K_{f} \sup _{-h \leq \theta \leq 0} \mathbb{E}|x(t-\theta)|^{2}
$$

and

$$
\mathbb{E}|g(t)|^{2} \leq K_{g} \sup _{-h \leq \theta \leq 0} \mathbb{E}|x(t-\theta)|^{2}
$$

for all $t \geq 0$. For any integer $k \geq 1$, by Hölder's inequality and Burkholder-Davis-Gundy inequality, one can derive that

$$
\begin{aligned}
& \mathbb{E}\left[\sup _{0 \leq \theta \leq h}|\eta(k h+\theta)|^{2}\right] \\
& \leq 3\left[\mathbb{E}|\eta(k h)|^{2}+h \int_{k h}^{(k+1) h} \mathbb{E}|f(s)|^{2} \mathrm{~d} s\right. \\
& \left.\quad+\mathbb{E}\left(\sup _{0 \leq \theta \leq h}\left|\int_{k h}^{k h+\theta} g(s) \mathrm{d} w(s)\right|\right)\right] \\
& \leq \beta_{h} e^{-k h \varepsilon},
\end{aligned}
$$


where $\beta_{h}=3 C_{h}\left(1+K_{f} h^{2} e^{h \varepsilon}+4 K_{g} h e^{h \varepsilon}\right)$. But, by Chebyshev's inequality, this implies

$$
\mathbb{P}\left\{\omega: \sup _{0 \leq \theta \leq h}|\eta(k h+\theta)|^{2}>e^{-(\varepsilon-\gamma) k h}\right\} \leq \beta_{h} e^{-\gamma k h} .
$$

By Borel-Cantelli lemma, there is a finite integer $k_{0}$ such that

$$
\sup _{0 \leq \theta \leq h}|\eta(k h+\theta)|^{2} \leq e^{-(\varepsilon-\gamma) k h} \quad \text { a.s. }
$$

for all $k \geq k_{0}$. Therefore, inequality (33) holds with $t_{h} \geq k_{0} h$.

Remark 1: From the proof of Theorem 1, it is observed that, letting $z^{T}(t)=\left[\eta^{T}(t) f^{T}(t) g^{T}(t)\right.$ $\frac{1}{h_{1}} \int_{t-h_{1}}^{t} f^{T}(s) \mathrm{d} s \frac{1}{h_{2}} \int_{t-h_{2}}^{t} f^{T}(s) \mathrm{d} s \int_{t-h_{1}}^{t} g^{T}(s) \mathrm{d} w(s)$ $\int_{t-h_{2}}^{t} g^{T}(s) \mathrm{d} w(s) x^{T}\left(t-h_{1}\right) x^{T}\left(t-2 h_{1}\right) C^{T} x^{T}\left(t-h_{1}-\right.$ $\left.\left.h_{2}\right) C^{T}\right]^{T}$, we can have a corollary derived from Theorem 1 with

$$
\left[\begin{array}{cc}
-T_{i} & C^{T} W_{i} \\
W_{i} C & -W_{i}
\end{array}\right] \leq 0, \quad i=1,2
$$

where $W_{i}>0$. This corollary can be easily applied to problems of stabilization by the approach of LMIs.

\section{EXAMPLES}

Example 1. Let us look at the following neutral stochastic delay system

$$
\begin{array}{r}
\mathrm{d}[x(t)-C x(t-h)]=\left[A_{0} x(t)+A_{1} x(t-h)\right] \mathrm{d} t \\
+\left[H_{0} x(t)+H_{1} x(t-h)\right] \mathrm{d} w(s)
\end{array}
$$

with

$$
\begin{aligned}
& C=\left[\begin{array}{cc}
-0.2 & 0 \\
1 & 0.2
\end{array}\right], A_{0}=\left[\begin{array}{cc}
0.5 & 0 \\
0 & 0.3
\end{array}\right], A_{1}=\left[\begin{array}{cc}
-1 & 0 \\
-1 & -1
\end{array}\right], \\
& H_{0}=\left[\begin{array}{cc}
0.2 & 0 \\
0 & 0.2
\end{array}\right], H_{1}=\left[\begin{array}{cc}
0.3 & 0 \\
0 & 0.3
\end{array}\right] .
\end{aligned}
$$

It is easy to verify that the existing results (see [2], [10], [12][17]) do not work. But, by Theorem 1, the upper bounds of time delay for exponential stability of system (36) is $h_{\max }=$ 0.35 .

Example 2. Deterministic systems may be regarded a special class of stochastic systems, e.g., the following deterministic neutral system is exactly system (1) with $A_{0}=A, A_{1}=B$ and $A_{2}=H_{0}=H_{1}=H_{2}=0$, i.e.,

$$
\dot{x}(t)-C \dot{x}(t-h)=A x(t)+B x(t-h)
$$

for all $t \geq 0$, where

$A=\left[\begin{array}{cc}-0.9 & 0.2 \\ 0.1 & -0.9\end{array}\right], B=-\left[\begin{array}{cc}1.1 & 0.2 \\ 0.1 & 1.1\end{array}\right], C=\left[\begin{array}{cc}-0.2 & \gamma \\ 0.2 & -0.1\end{array}\right]$

and $\gamma$ is a constant real number.

The case of $\gamma=0$ has been studied by many works (see, e.g., [4], [8] and [11]). However, results of [2], [4], [9], [11] and [18] are not (conveniently) applicable when $|\gamma| \geq 1$. For $\gamma \geq 2$, the criterion in [5] does not work, but the upper bounds $h_{\max }$ for exponential stability of (37) by other methods are listed in Table I, which shows that the results obtained by the methods proposed in this paper are less conservative in these cases.

Table I : $h_{\max }$ by different methods

\begin{tabular}{|c|c|c|c|}
\hline & $\gamma=2.0$ & $\gamma=2.2$ & $\gamma=2.4$ \\
\hline$[3]$ & 0.29 & 0.25 & 0.21 \\
\hline$[8]$ & 0.40 & 0.32 & 0.25 \\
\hline Theorem 1 & 0.46 & 0.39 & 0.30 \\
\hline
\end{tabular}

\section{CONCLUSION}

In this paper, delay-dependent criterion for stability of neutral stochastic delay systems has been presented by approach of LMIs. Numerical examples have been given to verify the effectiveness of the method proposed in this paper. Particularly, Example 2 demonstrates that our result developed for stochastic systems is competitive even when it is specialized to the deterministic cases.

\section{REFERENCES}

[1] W.-H. Chen, Z.-H. Guan and X. Lu, Delay-dependent exponential stability of uncertain uncertain stochastic systems with multiple delays, Systems \& Control Letters, 54 (2005) 547-555.

[2] W.-H. Chen and W.X. Zheng, Stability analysis and $H_{\infty}$-control of delayed neutral stochastic systems with time-varying parameter uncertainties, Proceedings of the 45th IEEE conference on decision and control, San Diego, U.S.A., December 2006 (pp. 961-966).

[3] W.-H. Chen and W.X. Zheng, Delay-dependent robust stabilization for uncertain neutral systems with distributed delays, Automatica, 43 (2007) 95-104.

[4] E. Fridman, New Lyapunov-Krasovskii functionals for stability of linear redarded and neutral type systems, Systems and Control Letters, 43 (2001) 309-319.

[5] E. Fridman and U. Shaked, A Descriptor system approach to $\mathrm{H}_{\infty}$ control of linear time-delay systems, IEEE Trans. Automatic Control, 47 (2002) 253-270.

[6] K. Gu, An integral inequality in the stability problem of time-delay systems, Proceedings of the 39th IEEE conference on decision and control, Sydney, Australia, December 2000 (pp. 2850-2810).

[7] J.K. Hale, Theory of functional differential equations. New York, U.S.: Springer-Verlag; 1977.

[8] Q.-L. Han, A descriptor system approach to robust stability of uncertain neutral systems with discrete and distributed delays, Automatica, 40 (2004) 1791-1796.

[9] Y. He, Q.-G. Wang, C. Lin and M. Wu, Augmented Lyapunov functional and delay-dependent stability criteria for neutral systems, Int. J. Robust Nonlinear Control, published online 4 November 2005 in Wiley InterScience (www.interscience.wiley.com). DOI: 10.1002/rnc.1039.

[10] V.B. Kolmanovskii and A. Myshkis, Introduction to the theory and applications of functional differential equations. Dordrecht, Netherland: Kluwer Academic Publishers; 1999.

[11] C.-H. Lien, K.-W. Wu and J.-G. Hsieh, Stability conditions for a class of neutral systems with multiple time delays, J. Math. Anal. and Appl., 245 (2000) 20-27.

[12] Q. Luo, X. Mao and Y. Shen, New criteria on exponential stability of neutral stochastic differential equations, Systems and Control Letters, 55 (2006) 826-834.

[13] M.S. Mahmoud, Robust $H_{\infty}$ control of linear neutral systems, Automatica, 36 (2000) 757-764.

[14] X. Mao, Exponential stability in mean square of neutral stochastic differential functional equations, Systems and Control Letters, 26 (1995) 245-251.

[15] X. Mao, Razumikhin-type theorems on exponential stability of neutral stochastic functional differential equations, SIAM J. Math. Anal., 28 (1997a) 389-401.

[16] X. Mao, Stochastic differential equations and applications. Chichester, U.K.: Horwood Publishing; 1997b.

[17] X. Mao, Asymptotic properties of neutral stochastic differential delay equations, Stochastics and Stochastics Rep., 68 (2000) 273-295. 
[18] S.-I. Niculescu, (2000), Further remarks on delay-dependent stability of linear neutral systems. Proceedings of MTNS 2000, Perpigan, France.

[19] J.H. Park and S. Won, Stability analysis for neutral delay-differential systems, J. of the Franklin Institute, 337 (2000) 1-9.

[20] B. Xu, Stability robustness bounds for linear systems with multiple time-varying delayed perturbations, International J. Systems Science, 28 (1997) 1311-1317.

[21] S. Xu, P. Shi, Y. Chu and Y. Zou, Robust stochastic stabilization and $H_{\infty}$ control of uncertain neutral stochastic time-delay systems, J. Math. Anal. and Appl., 314 (2006) 1-16. 